\title{
Dominant Role of the ERAP1 Polymorphism R528K in Shaping the HLA-B27 Peptidome Through Differential Processing Determined by Multiple Peptide Residues
}

\author{
Alejandro Sanz-Bravo, ${ }^{1}$ José Campos, $^{2}$ Marina S. Mazariegos, ${ }^{3}$ and José A. López de Castro ${ }^{1}$
}

Objective. To characterize the alterations, as well as their mechanisms, induced in the HLA-B27-bound peptidome expressed in live cells by the natural ERAP1 polymorphisms predisposing to ankylosing spondylitis (AS): R528K and N575D/Q725R.

Methods. HLA-B*27:05-bound peptides were isolated from 3 human lymphoid cell lines expressing distinct ERAP1 variants differing at residues 528 and/or 575/725. The high-performance liquid chromatographyfractionated peptide pools were compared by mass spectrometry based on identity of molecular mass and chromatographic retention time. The relative amount of each shared peptide in any given cell line pair was estimated from the respective ion peak intensities. Peptide sequencing was also carried out by mass spectrometry.

Results. HLA-B27-bound ligands predominant in the context of the ERAP1 variant with K528 collectively showed higher molecular mass, higher frequency of N-terminal residues resistant to ERAP1, and bulkier residues downstream of the $\mathrm{N}$-terminus, relative to peptides predominant in the $\mathrm{R528}$ context. None of

Supported by the Plan Nacional de $\mathrm{I}+\mathrm{D}+\mathrm{I}$ (grant SAF 2011/25681 to Dr. López de Castro), the Instituto de Salud Carlos III (RIER grant RD08/0075 to Dr. López de Castro), and the Fundación Ramón Areces (institutional grant to the Centro de Biología Molecular Severo Ochoa). Dr. Campos' work was supported by the Instituto de Salud Carlos III (grant PI11/004000). Ms Mazariegos' work was supported by the Plan Nacional de I+D+I (grant SAF 2011-24235).

${ }^{1}$ Alejandro Sanz-Bravo, MSc, José A. López de Castro, PhD: Centro de Biología Molecular Severo Ochoa, CSIC, and Universidad Autónoma, Madrid, Spain; ${ }^{2}$ José Campos, MD: Hospital Universitario Puerta de Hierro Majadahonda, Madrid, Spain; ${ }^{3}$ Marina S. Mazariegos, MSc: Hospital 12 de Octubre Health Research Institute and Universidad Complutense de Madrid, Madrid, Spain.

Address correspondence to José A. López de Castro, $\mathrm{PhD}$, Centro de Biología Molecular Severo Ochoa, Calle Nicolás Cabrera No. 1, Universidad Autónoma, 28049 Madrid, Spain. E-mail: aldecastro@cbm.csic.es.

Submitted for publication July 17, 2014; accepted in revised form November 25, 2014. these differences were observed with ERAP1 variants differing at positions $575 / 725$, but not at residue 528 . Neither R528K nor N575D/Q725R altered the mean length of $B * 27: 05$-bound ligands.

Conclusion. The R528K, but not the N575D/ Q725R, polymorphism alters the expression levels of many HLA-B*27:05-bound peptides, depending on the susceptibility of their $\mathrm{N}$-terminal residues to trimming and depending on the size of the amino acid side chains at multiple positions downstream of the N-terminus. The significant alterations in the $B * 27: 05$ peptidome and the structural features of the peptides that determine their differential expression in distinct ERAP1 contexts account for the association of the R528K polymorphism with AS.

Endoplasmic reticulum aminopeptidase 1 (ERAP1) is one of the main non-major histocompatibility complex (non-MHC) susceptibility factors in ankylosing spondylitis (AS) among individuals positive for HLA-B27 (1,2), accounting for $26 \%$ of the populationattributable risk (3). ERAP1 trims peptides in the endoplasmic reticulum to the optimal length, 9-10 residues, for MHC class I binding (4,5). A unique property of this molecule is that its activity is strongly dependent on substrate length, so that trimming essentially stops when the peptide reaches 8 residues (6). Crystallographic studies $(7,8)$ revealed that this feature is due to the fact that the peptide substrate binds in a cavity that is spatially distinct from the catalytic site. Occupancy of the substrate-binding site induces a conformational rearrangement that is critical for peptide trimming. Thus, ERAP1 polymorphisms can affect enzymatic activity by altering the catalytic site, the substrate-binding site, or the conformational transition to the active state.

The epistatic association of ERAP1 and HLAB27 in AS, which also holds for other MHC class I-associated diseases $(9,10)$, together with the observa- 
tion that AS-associated ERAP1 polymorphisms affect the enzymatic activity (for review, see ref. 11), suggest that the association of ERAP1 with AS is due to its effects on the HLA-B27 peptidome. The consequences of these effects are not necessarily limited to altering antigen presentation, since large changes in the peptidome may affect other features of HLA-B27, such as folding and stability, which are also peptide-dependent and potentially pathogenic (12). Indeed, AS-associated ERAP1 polymorphism was shown to substantially alter the peptidome and thermostability of $B^{*} 27: 04$ (13). That study addressed the joint effect of multiple co-occurring changes, but not of individual ones. The complex influence of the natural ERAP1 haplotypes on peptide processing was revealed in a recent study in which several ERAP1 variants were compared for their trimming of N-terminally extended precursors of a model peptide (14). Yet, genetic studies showed that the association of ERAP1 with AS fits a 2-mutation model in which both K528R and D575N/R725Q protect from AS. Among HLA-B27-positive individuals, those homozygous for the protective ERAP1 variants at both positions are 3-4 times less susceptible to AS than those carrying the corresponding predisposing alleles (2).

In the present study, we aimed to determine the effects of these AS-associated ERAP1 polymorphisms on the constitutive $\mathrm{B}^{*}$ 27:05 peptidome in live cells. Our strategy focused on alterations in peptide amounts occurring in distinct ERAP1 contexts, rather than on the presence or absence of specific epitopes, since failure to detect a peptide is not proof of its absence. Through an appropriate choice of the ERAP1 background of B*27: 05-positive cell lines, we separately assessed the role of the K528R and D575N/R725Q changes on shaping the $\mathrm{B}^{*}$ 27:05 peptidome. The mechanism underlying these effects was determined by comparing the structural features of the peptides presented in the various ERAP1 contexts.

\section{MATERIALS AND METHODS}

Cell lines. P50 (HLA-A*23, *24, B*27:05, *14, $\left.\mathrm{C}^{*} 02,{ }^{*} 08\right)$ and LG2 (HLA-A*02, B*27:05, C*01) are EpsteinBarr virus (EBV)-transformed lymphoblastoid cell lines. C1R$\mathrm{B}^{*}$ 27:05 (C1R05) is a transfectant of the lymphoid HLA class I-defective Hmy2.C1R cell line (15), expressing B*27:05 (16). Cells were cultured in RPMI 1640 medium supplemented with $2 \mathrm{~m} M$ L-glutamine and 10\% fetal bovine serum (Invitrogen).

Genomic sequencing and Western blotting. The sequence of ERAP1 in C1R cells has been previously reported (13). Exons 2-20 of ERAP1 in LG2 cells, encompassing the coding region of the mature protein, and the same exons (except for exons 3, 14, and 16) in P50 cells were separately amplified via polymerase chain reaction and sequenced using the same procedure. For ERAP2, the AS-associated polymorphisms at codon 392 (rs2549782, exon 7) and at the exon 10 splice site (rs2248374), which are in strong linkage disequilibrium, were determined by genomic sequencing. The latter polymorphism affects ERAP2 expression (17). The rs9901869 polymorphism, which was used to establish AS association of the NPEPPS gene, which codes for the puromycin-sensitive aminopeptidase (18), was also sequenced. ERAP1 and ERAP2 protein levels were determined by Western blotting of whole lysates of $5 \times 10^{5}$ cells as described elsewhere (13), except that electroblotting was performed at $80 \mathrm{~V}$ for 80 minutes.

Isolation of HLA-B27-bound peptides. HLA-B27bound peptides were isolated as previously described (19). Briefly, $\sim 10^{10}$ cells were lysed in $1 \%$ Igepal CA-630 with a mixture of protease inhibitors (Roche). The soluble fraction was subjected to affinity chromatography using ME1 (IgG1), an anti-HLA-B7/B27/B22 monoclonal antibody that recognizes the bulk of HLA-B27-peptide complexes (20). HLAB27-bound peptides were eluted with $0.1 \%$ trifluoroacetic acid, filtered, concentrated, and subjected to fractionation by high-performance liquid chromatography (HPLC) at a flow rate of $100 \mu \mathrm{l} /$ minute, as described elsewhere (21). Fractions of $50 \mu \mathrm{l}$ were collected and stored at $-20^{\circ} \mathrm{C}$.

Mass spectrometry (MS). Analysis of individual HPLC fractions by matrix-assisted laser desorption ionization-timeof-flight (MALDI-TOF) MS and peptide sequencing of the most prominent ion peaks in each fraction by MALDI-TOF/ TOF MS/MS were carried out using a 4800 Proteomics Analyzer (Applied Biosystems) as described elsewhere (22). The MALDI-TOF MS spectra were acquired in reflector-positive mode at $25 \mathrm{kV}$ in the mass-to-charge $(\mathrm{m} / \mathrm{z})$ range of $800-2000$, using a signal-to-noise ratio cutoff of 3 , and were processed using Data Explorer software version 4.9 (Applied Biosystems). Sample handling and acquisition parameters were tightly controlled to minimize differences among the samples to be compared in each experiment. Automated comparison of the MALDI-TOF MS spectra corresponding to correlative HPLC fractions from different cell lines was performed using MShandler software as previously described (13).

Susceptibility of N-terminal residues to ERAP1 trimming. We estimated the susceptibility of $\mathrm{N}$-terminal residues to ERAP1 trimming by assigning a score ranging from 0 to 100 to each amino acid, based on a previously reported assay (23), as described elsewhere (13). Proline and any residue immediately preceding proline were assigned a score of 0 .

\section{RESULTS}

Expression of ERAP1/ERAP2 variants in B*27: 05-positive cell lines. The ERAP1 variant in LG2 showed 5 AS-predisposing polymorphisms, whereas the one in P50 had the corresponding protective changes. The ERAP1 variant in C1R05 carried 3 AS-associated changes (at positions 349,575 , and 725 ), and 2 protective changes (at positions 127 and 528). All 3 cell lines had the AS-protective residue E730 and were homozygous at all of the polymorphic ERAP1 and ERAP2 positions, 
Table 1. ERAP1 and ERAP2 polymorphism in B*27:05-positive cell lines*

\begin{tabular}{|c|c|c|c|c|c|c|}
\hline \multirow[b]{2}{*}{ SNP } & \multirow[b]{2}{*}{ Exon } & \multirow{2}{*}{$\begin{array}{l}\text { Nucleotide/ } \\
\text { aa residue }\end{array}$} & \multirow{2}{*}{$\begin{array}{l}\text { Consensus } \\
\text { sequence }\end{array}$} & \multicolumn{3}{|c|}{ Cell line } \\
\hline & & & & LG2 & $\mathrm{C} 1 \mathrm{R}$ & P50 \\
\hline \multicolumn{7}{|l|}{ ERAP1 } \\
\hline rs 26653 & 2 & $380 / 127$ & $\mathrm{~g} / \mathrm{R} \dagger$ & $\mathrm{g} / \mathrm{R} \dagger$ & $\mathrm{c} / \mathrm{P}$ & $\mathrm{c} / \mathrm{P}$ \\
\hline rs26618 & 5 & $828 / 276$ & $\mathrm{a} / \mathrm{I}$ & $\mathrm{a} / \mathrm{I}$ & $\mathrm{g} / \mathrm{M}$ & $\mathrm{a} / \mathrm{I}$ \\
\hline rs 27895 & 6 & $1037 / 346$ & $\mathrm{~g} / \mathrm{G}$ & $\mathrm{g} / \mathrm{G}$ & $\mathrm{g} / \mathrm{G}$ & $\mathrm{g} / \mathrm{G}$ \\
\hline rs2287987 & 6 & $1045 / 349$ & $\mathrm{a} / \mathrm{M} \dagger$ & $\mathrm{a} / \mathrm{M} \dagger$ & $\mathrm{a} / \mathrm{M} \dagger$ & $\mathrm{g} / \mathrm{V}$ \\
\hline rs30187 & 11 & $1583 / 528$ & $\mathrm{a} / \mathrm{K} \dagger$ & $\mathrm{a} / \mathrm{K} \dagger$ & $\mathrm{g} / \mathrm{R}$ & $\mathrm{g} / \mathrm{R}$ \\
\hline rs10050860 & 12 & $1723 / 575$ & $\mathrm{~g} / \mathrm{D} \dagger$ & $\mathrm{g} / \mathrm{D} \dagger$ & $\mathrm{g} / \mathrm{D} \dagger$ & $\mathrm{a} / \mathrm{N}$ \\
\hline rs17482078 & 15 & $2174 / 725$ & $\mathrm{~g} / \mathrm{R} \dagger$ & $\mathrm{g} / \mathrm{R} \dagger$ & $\mathrm{g} / \mathrm{R} \dagger$ & $\mathrm{a} / \mathrm{Q}$ \\
\hline rs 27044 & 15 & $2188 / 730$ & $\mathrm{c} / \mathrm{Q} \dagger$ & $\mathrm{g} / \mathrm{E}$ & $\mathrm{g} / \mathrm{E}$ & $\mathrm{g} / \mathrm{E}$ \\
\hline \multicolumn{7}{|l|}{ ERAP2 } \\
\hline rs 2549782 & 7 & $1176 / 392$ & $\mathrm{~g} / \mathrm{K} \dagger$ & $\mathrm{g} / \mathrm{K}^{\dagger}$ & $\mathrm{g} / \mathrm{K}^{\dagger}$ & $\mathrm{g} \dagger, \mathrm{t} / \mathrm{K} \dagger, \mathrm{N}$ \\
\hline rs 2248374 & 10 & $1575 /-$ & $\mathrm{a} \dagger$ & $\mathrm{a} \dagger$ & $\mathrm{a} \dagger$ & $\mathrm{a} \uparrow, \mathrm{g}$ \\
\hline \multicolumn{7}{|l|}{ NPEPPS } \\
\hline rs9901869 & - & - & - & $\mathrm{g}$ & $\mathrm{g}$ & $\mathrm{g}$ \\
\hline
\end{tabular}

* Changes in the coding or forward strands are shown. The nucleotide/amino acid (aa) residue numbering and consensus sequence are from human ERAP1 isoform 2 (accession no. Q9NZ08-2) and ERAP2 isoform 1 (accession no. Q6P179-1). Single-nucleotide polymorphism (SNP) rs9901869 (a/g) is located in an intergenic region. All polymorphic positions were determined by genomic sequencing. The ERAP1 sequence from C1R cells has been previously reported (13) and is shown here for comparison only. Although genotyping of the ERAP2 polymorphism rs2549782 at codon 392 previously suggested heterozygocity of C1R (13), genomic sequencing in the present study revealed that this cell line is actually homozygous both at that SNP (gg) and at the linked one, rs2248374.

$\dagger$ SNP associated with an increased risk of ankylosing spondylitis $(3,18,24)$.

except for P50. They were genotypically concordant at the NPEPPS marker rs9901869 (Table 1).

ERAP1 protein expression was $\sim 2$-fold and 3 -fold higher in P50 and LG2 cells, respectively, than in C1R05 cells (Figure 1A). The high ERAP1 expression in LG2 reflects a general phenomenon whereby there is a consistently higher expression of ERAP1 variants carrying the AS-associated polymorphism K528 (24,25). ERAP2 protein levels were similar in the 3 cell lines (Figure 1B).

Dominant role of the K528R polymorphism in altering the $B * 27: 05$ peptidome. The $B * 27: 05$-bound peptide pools from LG2, P50, and C1R05 cells were isolated after immunopurification of HLA-B27 and acid extraction, were HPLC-fractionated under identical conditions and consecutive runs, and each fraction was analyzed by MALDI-TOF MS. The peptide pools were compared by matching the ion peaks with the same retention time and $\mathrm{m} / \mathrm{z}$. These were considered identical peptides and were confirmed in all cases in which such peaks were sequenced from distinct cell lines (see Supplementary Table 1, available on the Arthritis \& Rheumatology web site at http://onlinelibrary.wiley.com/doi/ 10.1002/art.38980/abstract).

Three pairwise comparisons were carried out: LG2/P50, LG2/C1R05, and C1R05/P50. The 2 former comparisons were aimed at determining the effect of 2 distinct combinations of ERAP1 polymorphisms, both including the K528R mismatch. C1R05/P50 allowed us to assess the effect of polymorphisms other than the one
A
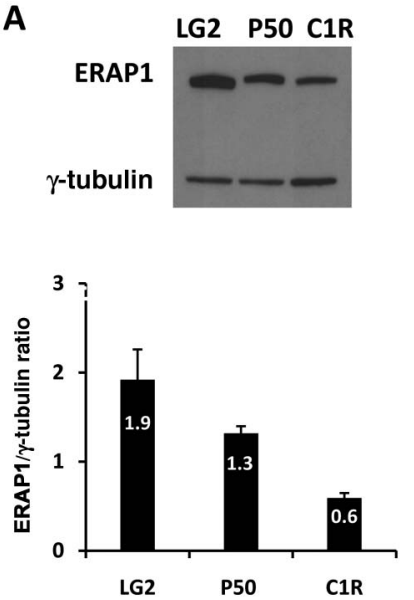

B
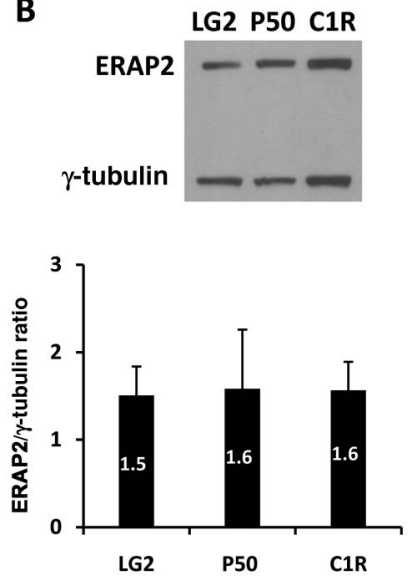

Figure 1. Western blot analysis of endoplasmic reticulum aminopeptidase 1 (ERAP1) and ERAP2 protein expression. The expression of ERAP1 (A) and ERAP2 (B) in the LG2, P50, and C1R cell lines was determined by Western blotting. $\gamma$-tubulin was used as a loading control. Results from a representative experiment (top) are shown, along with data from 3 independent analyses (bottom). Numbers inside the bars are the values represented by the bars. Values are the mean $\pm \mathrm{SD}$. 
A ALL SHARED PEPTIDES
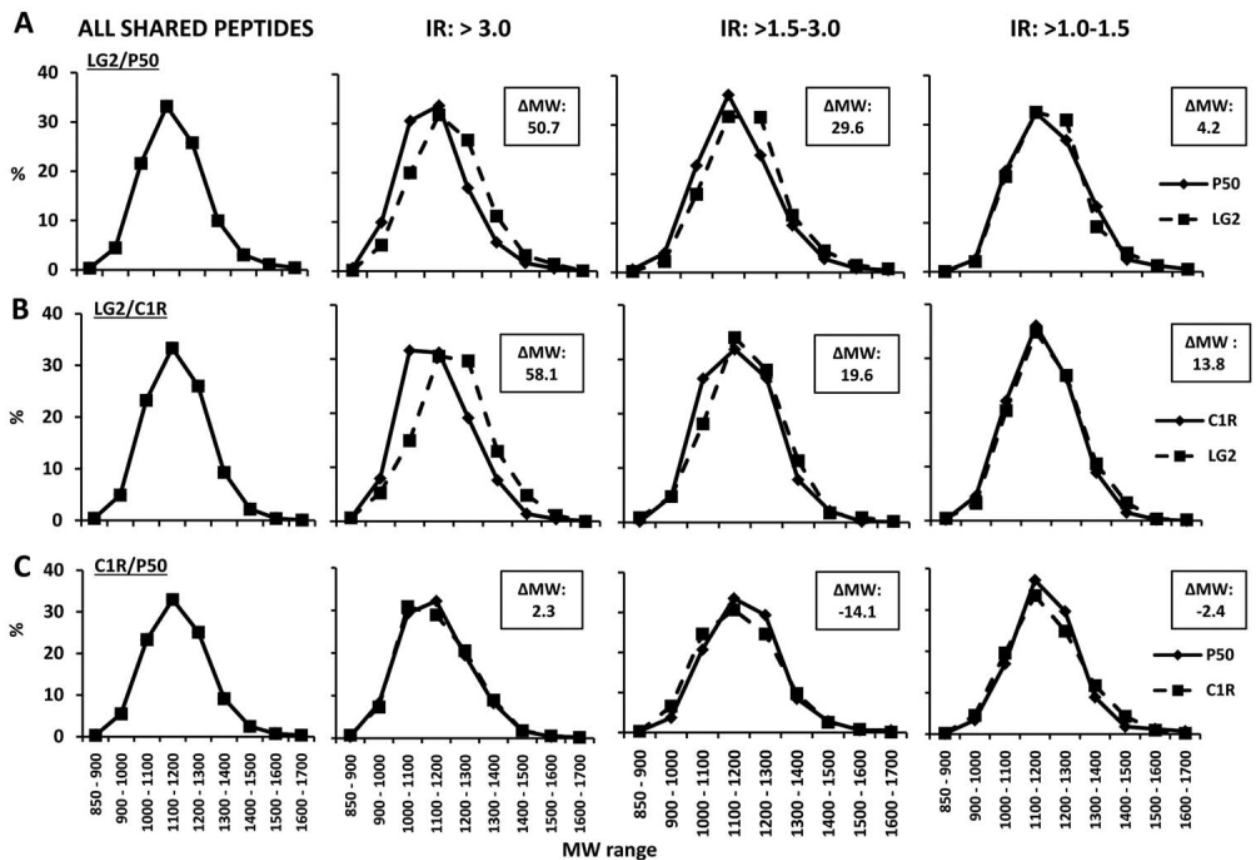

Figure 2. Size distribution of B*27:05 ligands as a function of their relative abundance in various ERAP1 contexts. Shared peptides were classified according to the intensity ratio (IR) of one cell line relative to the other: $>3$-fold, $>1.5-3$-fold, and $>1-1.5$-fold. Results for the LG2/P50 (A), the LG2/C1R05 (B), and the C1R05/P50 (C) cell line comparisons are shown. The left histogram shows the molecular weight distribution of shared ligands detected for the 2 cell lines (7,075, 6,953, and 6,204 respectively). The other 3 histograms compare the molecular weight distribution of the peptide sets by IR subgroup. The difference in the mean molecular weight $(\Delta \mathrm{MW})$ between the peptide subsets compared is indicated in each histogram. Data are from a representative experiment of those shown in Supplementary Table 2 (available on the Arthritis \& Rheumatology web site at http://onlinelibrary.wiley.com/doi/10.1002/art.38980/abstract).

at residue 528, including D575N/R725Q (Table 1). Each comparison was carried out 2 or 3 times. As a control, the $\mathrm{B}^{*} 27: 05$ peptide pools from 2 independent preparations of C1R05 cells were compared (see Supplementary Table 2, available on the Arthritis \& Rheumatology web site at http://onlinelibrary.wiley.com/doi/10.1002/ art.38980/abstract). The methodology used for these comparisons has been previously described (13).

Peptides shared among any 2 cell lines were classified according to the relative intensity of the corresponding ion peak in both cell lines, designated as the intensity ratio (IR), in 3 subsets: IR $>3$-fold, IR $>1.5$ 3 -fold, and IR $>1-1.5$-fold intensity in one cell line relative to the other. This was based on the assumption that the differential effects of ERAP1 polymorphism on peptide expression would be best revealed among peptides showing the largest disparity in their amounts between the cell lines being compared. The molecular weight distribution of the peptides in each subset from one cell line was compared with the corresponding subset in the other cell line (Figure 2). A total of $\sim 6,000-7,000$ shared peptides were detected in each comparison. They showed Gaussian molecular weight distributions with mean values of $\sim 1160-1180$ daltons in all cases. Two distinct patterns were observed.

In the LG2/P50 and LG2/C1R05 comparisons, the peptides with an IR of $>3$ in LG2 cells showed a shift in the molecular weight distribution curve toward larger values relative to the peptides with an IR of $>3$ in either the P50 or C1R05 cell lines (Figures 2A and B). The mean molecular weight of LG2-derived peptides in this subset was $\sim 52$ and 59 daltons higher than the corresponding values for P50 and C1R05, respectively. A more attenuated difference was observed for the peptide subsets with an IR of $>1.5-3$ in both comparisons, which was close to the background levels in the C1R05/C1R05 control (Supplementary Table 2, available on the Arthritis \& Rheumatology web site at http://onlinelibrary.wiley. com/doi/10.1002/art.38980/abstract). For peptides with similar IR (>1-1.5), virtually no differences were observed (Figures 2A and B). These results indicate that B*27:05-bound peptides predominate in LG2, relative to P50 or C1R05, and have higher molecular weights than those that predominate in any of these cell lines, relative 
to LG2. In contrast, no differences in molecular weight were observed between C1R05 and P50 for any of the IR ranges (Figure 2C and Supplementary Table 2, available on the Arthritis \& Rheumatology web site at http://online library.wiley.com/doi/10.1002/art.38980/abstract). That the differences were observed mainly in the IR $>3$ subsets suggests that many HLA-B27 ligands either bypass ERAP1 processing or both their generation and destruction are concomitantly affected by ERAP1 polymorphism, resulting in similar epitope amounts with distinct variants, as previously reported (26).

ERAP1 in LG2 differs from the P50 and C1R variants at residues 127 and 528, whereas the 3 latter cell lines are identical at both positions (Table 1). Thus, the 127 and/or 528 polymorphisms are responsible for the molecular weight differences observed. Since K528R, but not $\mathrm{R} 127 \mathrm{P}$, is known to affect ERAP1 activity (27), most of the alterations in the $\mathrm{B}^{*} 27: 05$ peptidome between LG2 and P50 or C1R05 are probably due to residue 528. In contrast, the 4 amino acid differences between the ERAP1 variants of C1R and P50 (Table 1) had no detectable effect on the MW of the $B^{* 27: 05 ~ l i g a n d s . ~}$

$\mathrm{N}$-terminal residues of HLA-B27 ligands as determinants of the effect of ERAP1 variants on the $B * 27: 05$ bound peptidome. If the alterations in the $\mathrm{B}^{*} 27: 05$ peptidome described above are due to the different enzymatic activity of ERAP-1 in each cell line, one would expect that those peptides predominant in cells expressing distinct ERAP1 variants may differ in the susceptibility of their flanking and/or P1 residues to trimming. Thus, for each pairwise comparison, the sequence of $\sim 50-100$ peptides from each IR subset of both cell lines was obtained, up to a total of 470 nonredundant sequences (Supplementary Table 1, available on the Arthritis \& Rheumatology web site at http://onlinelibrary.wiley.com/doi/10.1002/art.38980/ abstract), of which 296 were previously known HLA-B27 ligands (28).

For these analyses, the peptides were subdivided into 2 groups: those with an IR of $>3$ and those with an IR of $>1-3$. The susceptibility of the $2 \mathrm{~N}$-terminal flanking residues (P-2 and $\mathrm{P}-1)$ and the N-terminal residue of the natural ligand (P1) to ERAP1 was computed by assigning a score ranging from 0 to 100 to each residue, based on its cleavage efficiency. The mean scores for peptides within each subset at P-2, P-1, and P1 were compared (Table 2). The mean susceptibility of the flanking residues to ERAP1 observed in the 3 pairwise comparisons was very similar regardless of the IR subset being considered. Moreover, no differences in the mean susceptibility of $\mathrm{P} 1$ residues were observed in the C1R05/P50 comparison. In contrast, in LG2/P50 and
Table 2. Susceptibility of N-terminal flanking and P1 residues of B*27:05 ligands to ERAP1

\begin{tabular}{lcccc}
\hline & \multicolumn{3}{c}{ ERAP1 susceptibility score $\dagger$} \\
\cline { 2 - 5 } Comparison, & & \multicolumn{3}{c}{ P-2 +} \\
peptides* & $\mathrm{P}-2$ & $\mathrm{P}-1$ & $\mathrm{P}-1$ & $\mathrm{P} 1$ \\
\hline P50/LG2 & & & & \\
IR $>3$ & & & & \\
70/49 & $24.1 / 28.3$ & $53.5 / 42.8$ & $77.5 / 70.1$ & $27.9 / 16.5$ \\
$\quad$ Mean score ratio & 0.9 & 1.3 & 1.1 & $1.7 \ddagger$ \\
IR $>1-3$ & & & & \\
57/62 & $28.5 / 34.1$ & $47.2 / 45.2$ & $74.2 / 78.5$ & $24.1 / 19.8$ \\
Mean score ratio & 0.8 & 1.0 & 1.0 & 1.2 \\
C1R05/LG2 & & & & \\
IR $>3$ & & & & \\
101/52 & $37.5 / 34.9$ & $42.5 / 43.3$ & $78.9 / 78.2$ & $28.4 / 17.0$ \\
Mean score ratio & 1.1 & 1.0 & 1.0 & $1.7 \ddagger$ \\
IR $>1-3$ & & & & \\
50/52 & $30.3 / 37.4$ & $46.6 / 40.9$ & $76.3 / 78.3$ & $26.4 / 23.4$ \\
Ratio & 0.8 & 1.1 & 1.0 & 1.1 \\
P50/C1R05 & & & & \\
IR $>3$ & & & & \\
74/63 & $36.1 / 34.6$ & $41.9 / 39.6$ & $77.2 / 73.3$ & $29.7 / 29.0$ \\
Mean score ratio & 1.0 & 1.1 & 1.1 & 1.0 \\
IR $>1-3$ & & & & \\
76/61 & $31.5 / 37.5$ & $46.2 / 47.8$ & $77.1 / 84.1$ & $23.5 / 26.6$ \\
Mean score ratio & 0.8 & 1.0 & 0.9 & 0.9 \\
\hline
\end{tabular}

* Peptides shared among any 2 cell lines were classified according to the relative intensity of the corresponding ion peak in both cell lines. This was designated as the intensity ratio (IR), which is categorized here as $>3$-fold or $>1-3$-fold. Shown are the number of peptides sequenced from the indicated subset in each cell line.

$\dagger$ Mean susceptibility score for the corresponding residues at the indicated positions in each peptide subset of the 2 cell lines being compared: the $2 \mathrm{~N}$-terminal flanking residues (P-2 and P-1) and the $\mathrm{N}$-terminal residue of the natural ligand (P1) of ERAP1.

$\ddagger P<0.05$ by chi-square test, between the susceptibility scores of the corresponding peptides.

LG2/C1R05, a higher susceptibility of the P1 residues from P50- or C1R05-derived peptides relative to LG2 was observed when the IR $>3$ subsets were compared, but not among peptides with similar abundance (IR $>1-3)$.

Thus, we analyzed in more detail the frequency of P1 residues, based on their susceptibility to ERAP1. For each pairwise comparison, the joint frequencies of $\mathrm{P} 1$ residues with high (score of $\geq 50$ ), intermediate (score of 21-49), or low (score of 0-20) susceptibility in each peptide subset were compared (Figure 3 ). The peptides from the IR $>3$ subsets in LG2/P50 and LG2/C1R05 showed statistically significant differences in the usage of $\mathrm{P} 1$ residues with intermediate and low scores, but not high scores (Figures $3 \mathrm{~A}$ and $\mathrm{B}$ ). P1 residues with intermediate susceptibility were less abundant, and those with low susceptibility more abundant, among those peptides predominant (IR >3) in the context of the most active ERAP1 variant (LG2), relative to the less active 

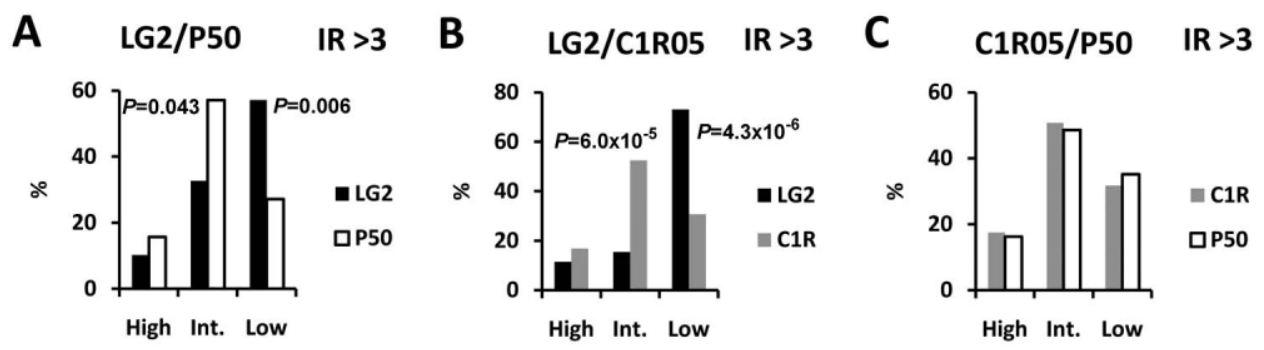

IR $>1-3$

IR $>$ 1-3

IR $>1-3$
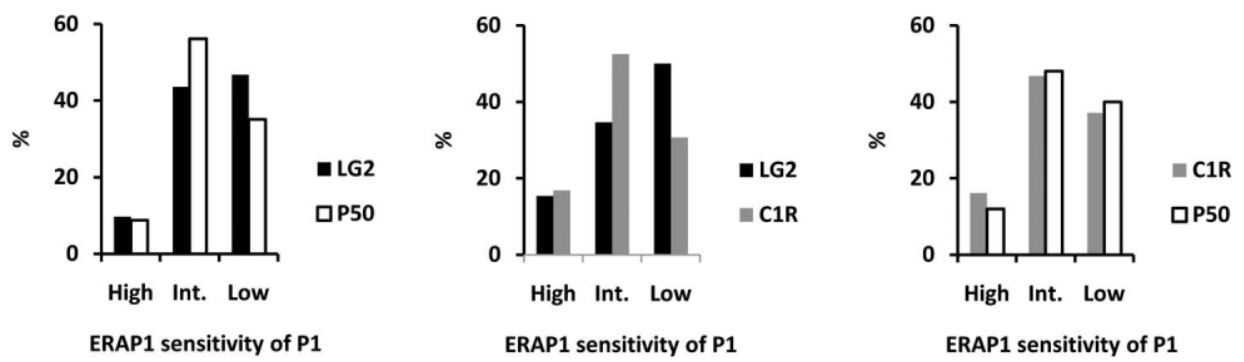

Figure 3. ERAP1 susceptibility of the $\mathrm{P} 1$ residues from $\mathrm{B}$ *27:05 ligands showing differential abundance among the cell lines studied. The frequency of P1 residues with high (score of $\geq 50 ; \mathrm{A}, \mathrm{C}, \mathrm{L}, \mathrm{M}$, and Y), intermediate (Int.) (score of 21-49; F, G, H, I, N, Q, S, and T), and low (score of 0-20; D, E, K, P, R, V, and W) susceptibility to ERAP1 trimming among the peptides sequenced from LG2 and P50 (A), LG2 and C1R05 (B), and C1R05 and P50 (C) from their corresponding intensity ratio (IR) subset ( $>3$ [top] or $>1-3$ [bottom]) in the corresponding pairwise comparison was determined. Statistically significant $(P<0.005)$ differences among the individual residues, as determined by chi-square test, are specified.

ones (P50 and C1R05). The same tendency was observed among peptides with an IR of $>1-3$ in both comparisons, although these differences did not reach statistical significance. No difference in the susceptibility of P1 residues to ERAP1 was observed in the C1R05/ P50 comparison (Figure 3C).

These results indicate that the ERAP1 polymorphism K528R alters the B*27:05-bound peptidome by regulating epitope destruction as a function of the susceptibility of the P1 residues of HLA-B27 ligands to trimming. The observed patterns suggest that highly susceptible residues are efficiently trimmed by all ERAP1 variants, leading to a similar destruction of the corresponding epitopes. P1 residues with intermediate susceptibility are more efficiently cleaved by the most active variant, resulting in their higher frequency among the predominant peptides in a less active ERAP1 context. The increased frequency of ERAP1-resistant P1 residues among HLA-B27 ligands that are predominant in a more active context is the logical consequence of the higher destruction of peptides with more susceptible P1 residues, together with limited digestion of resistant ones even by the most active variant.
Little influence of the differential activity of ERAP1 variants on the length of HLA-B27 ligands. The differences in molecular weight among B*27:05 ligands predominant in distinct ERAP1 contexts could be due to differential residue usage, differential length, or both. Thus, we compared the mean molecular weight and length distributions of the peptides sequenced from the IR $>3$ subsets in the LG2/P50 and LG2/C1R05 comparisons. In LG2/P50, the predominant peptides sequenced from LG2 ( $\mathrm{n}=49$; mean MW 1169.4 daltons and mean length 9.5 residues) and those from P50 $(\mathrm{n}=70$; mean MW 1094.5 daltons and mean length 9.3 residues) showed a $\Delta \mathrm{MW}$ of 74.9 daltons. For LG2/C1R05, the predominant peptides sequenced from LG2 $(\mathrm{n}=52$; mean MW 1195.7 daltons and mean length 9.6 residues) and those from C1R05 $(\mathrm{n}=101$; mean MW 1118.9 daltons and mean length 9.3) showed a $\Delta \mathrm{MW}$ of 76.8 daltons. These differences were only slightly higher than those observed in the global peptide comparisons (Figure 2 and Supplementary Table 2, available on the Arthritis \& Rheumatology web site at http:/online library.wiley.com/doi/10.1002/art.38980/abstract), suggesting that the sequenced peptides are representative 


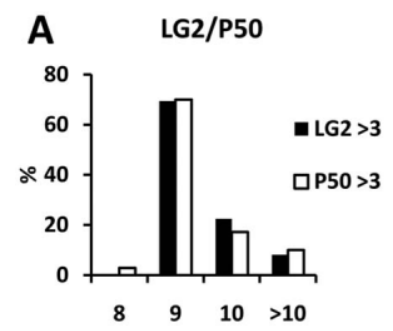

LG2/C1R05

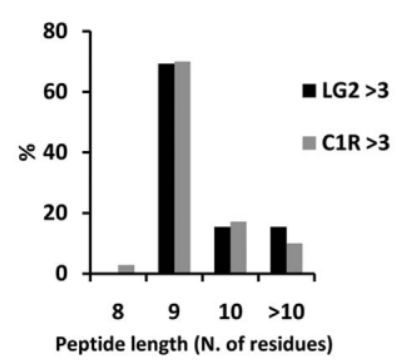

B

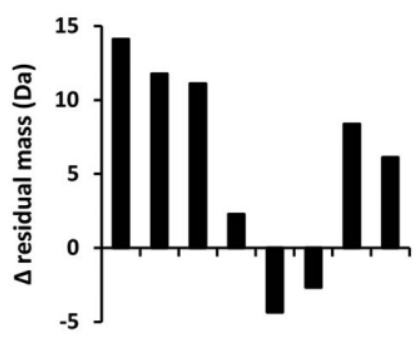

LG2/C1R05 (IR>3)

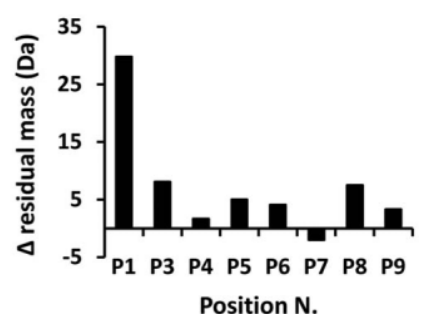

Position $\mathrm{N}$.

Figure 4. Length distribution and residual mass differences among B*27:05 ligands showing differential abundance among the cell lines studied. A, Length distribution among the sequenced peptides predominant (intensity ratio [IR] >3) in LG2 and P50 (top) or LG2 and C1R05 (bottom) in the corresponding pairwise comparisons. B, Mean differential mass ( $\Delta$ residual mass) at the indicated positions between residues of the $\mathrm{B}^{*} 27: 05$ ligands predominant (IR $>3$ ) in LG2 and P50 (top) or LG2 and C1R05 (bottom) in the corresponding pairwise comparisons.

of their respective IR subsets. Yet, they showed very similar length distribution (Figure 4A).

The results indicate that the molecular weight differences among $B^{*} 27: 05$ ligands are due to differential residue usage and not to distinct peptide length. As expected, in C1R05/P50, the predominant peptides sequenced from C1R05 ( $\mathrm{n}=63$; mean MW 1127.1 daltons and mean length 9.5 residues) or P50 ( $=74$; mean MW 1134.4 daltons and mean length 9.6 residues) showed essentially no differences in either mean molecular weight or length.

Influence of residues downstream of the $\mathrm{N}$-terminus on the processing of HLA-B27 ligands in an ERAP1 variant-dependent way. To determine the structural basis for the observed molecular weight differences, we selected the 9-mers from the IR $>3$ subsets in the LG2/P50 and LG2/C1R05 comparisons, since they accounted for $\sim 70 \%$ of the sequenced peptides from each subset (Figure 4A). The mean molecular weight between the predominant 9-mers sequenced from LG2 (1107.6 daltons) and P50 (1060.8 daltons) was 46.8 daltons higher for LG2. This was due to moderate

differences in the mean residue mass along multiple positions, mainly P1, P3, P4, P8, and P9 (Figure 4B). For LG2/C1R05, the mean molecular weight between the predominant 9-mers sequenced from LG2 (1138.8 daltons) and C1R05 (1081.5 daltons) was 57.3 daltons higher in LG2. Once again, this difference was due to the contribution of multiple positions, but now P1 ( $\Delta$ residual mass 29.8 daltons) accounted for $52 \%$ of the total. In C1R05/P50, the predominant 9-mers showed essentially no difference in their mean molecular weight: 1070.7 daltons and 1071.7 daltons, respectively.

Thus, the P1 residue only partially accounts for the molecular weight differences induced in the HLAB27 peptidome by ERAP1 variants. Our results reveal an additional contribution of the sequence downstream of the N-terminus on the shaping of the peptidome by ERAP1 allotypes differing at K528R. An overall tendency toward bulkier residues at multiple positions was observed among HLA-B27 ligands predominant in the most active ERAP1 context (K528) of LG2 cells.

\section{DISCUSSION}

In vitro studies with ERAP1 mutants demonstrated that the disease-protective changes R528 $(2,8,14,26,27,29)$ and Q725 $(2,14)$ decreased the enzymatic activity, whereas N575 increased ERAP1 activity in 2 studies $(14,26)$, but had no effect in others in which either 1 substrate (2) or 2 substrates (27) were examined. This apparent discrepancy may be due to the fact that the effect of a given polymorphism on peptide trimming is dependent on both its structural context and the substrate tested. By appropriate choice of the ERAP1/ ERAP2 background of $3 \mathrm{~B} * 27: 05$-positive cell lines, we analyzed the effect of the K528R mutation in the presence (LG2/P50) or absence (LG2/C1R05) of the D575N/R725Q changes on the $\mathrm{B} * 27: 05$ peptidome. In addition, the P50/C1R05 comparison allowed us to address the effect of D575N/R725Q in the absence of polymorphism at residue 528 .

This study did not address the presence or absence of specific ligands in a variant-dependent way, since MS analyses cannot formally establish the absence of a peptide. Our purpose was to define global effects on the amount of shared peptides as a function of ERAP1 polymorphism, since quantitative changes may significantly affect the biologic behavior of HLA-B27. Specific HLA-B27 epitopes produced only in particular ERAP1 contexts are likely to exist and might be relevant for AS, but these should be investigated separately.

The ME1 antibody that was used to purify HLA- 
B27 recognizes the bulk of B27-peptide complexes. Although it is formally impossible to exclude the possibility that some HLA-B27 ligands might be overlooked with this or any other antibody, there is no evidence that ME1 might be reacting with a particular fraction of the peptidome. In a previous study, in which both ME1 and the anti-MHC class I antibody W6/32 were used, no significant differences in the length or $\mathrm{P} 1$ residue frequencies of the HLA-B27 ligands were observed (30).

Our experimental approach was based on MALDI-TOF MS. The use of this technique for comparing MHC class I-bound peptidomes has been discussed in detail elsewhere (13). It will only be emphasized here that it is a powerful and reproducible way to reveal collective tendencies within large sets of peptides. This was clear from the reproducibility of the molecular weight distribution patterns among subsets of HLA-B27 ligands, which were classified according to their relative ion peak intensities, across individual experiments. Yet, MALDI-TOF MS is not quantitative and cannot be applied to quantify differences in expression on an individual peptide basis.

Our results revealed that in the cell lines examined, the AS-protective K528R mutation had a substantial effect on the $B^{*} 27: 05$ peptidome that was independent of residues 575/725. In addition, in the absence of polymorphism at residue 528 , a mismatch at $575 / 725$ had little effect. Since natural variants contain multiple changes in strong linkage disequilibrium, K528/R127 and R528/P127 co-occurred in our cell lines. Thus, although we cannot unambiguously assign to one of these mutations the observed peptide differences between LG2 and C1R05 or P50, it is very likely that they are mainly due to K528R, since R127P has little effect on ERAP1 activity (27). D575N/R725Q co-occurred with M349V in P50. In one study (14), M349V/D575N/ R725Q did not alter ERAP1 activity, which is consistent with the absence of significant peptidome alterations across these 3 differences in C1R05/P50. The same study reported that in the context of M349, D575N/R725Q decreased ERAP1 activity. Thus, the possibility that in the absence of $\mathrm{M} 349 \mathrm{~V}$, the $\mathrm{D} 575 \mathrm{~N} / \mathrm{R} 725 \mathrm{Q}$ changes might have a larger effect on the HLA-B27 peptidome cannot be ruled out.

Since the two cell lines carrying R528 differed $\sim 2$-fold in the expression levels of ERAP1, we cannot exclude the possibility that higher expression on P50 relative to C1R05 might obscure a potentially higher activity of the ERAP1 variant in the latter cell line, which carries the AS-predisposing residues D575/R725. This possibility would also explain the finding that LG2 showed comparable differences in the B27-bound peptidome with both cell lines, since a putatively higher activity of the ERAP1 variant in C1R05 might be compensated by its lower expression. A differential influence of ERAP2 in our cell lines can be excluded, since all expressed the K392 variant and showed similar enzyme levels. A contribution of puromycin-sensitive aminopeptidase, an enzyme involved in postproteasomal processing, to the observed peptide differences is also unlikely based on concordance of the 3 cell lines at the AS-associated marker rs9901869.

An interesting issue concerns the higher expression of ERAP1 in LG2 (K528) relative to that in C1R05 and P50 (R528). A correlation between the strength of the single-nucleotide polymorphism association with AS, including rs30187 (coding for residue 528), and the strength of the association with ERAP1 transcript abundance has been reported (24). Constantino and colleagues (25) subsequently observed that the risk haplotype of ERAP1 in AS, including K528, is associated with higher ERAP1 protein expression in EBV-transformed cell lines, which we confirmed in an independent panel of HLA-B27-positive and HLA-B27-negative cell lines (Martin-Esteban A, et al: unpublished observations). Higher expression of the most active ERAP1 variants may be a mechanism by which to enhance the effect of increased enzymatic activity induced by an ASpredisposing polymorphism.

Peptide sequencing revealed the basis for the alterations in the $B * 27: 05$ peptidome induced by the ERAP1 polymorphism. The higher frequency of ERAP1-resistant P1 residues among predominant ligands in LG2 (K528), relative to those predominant in less active (R528) contexts, reflects a larger destruction of ligands with ERAP1-sensitive P1 residues by the more active variant. This was shown by increased expression of these peptides in the less active contexts (C1R05 and P50). Seregin et al (31) recently noted that highly active ERAP1 variants carrying AS-predisposing polymorphisms, including K528, led to reduced expression of multiple HLA-B27 ligands due to increased epitope destruction, which is consistent with our results. Yet, as shown here, ERAP1 variants with K528 also induced the increased expression of other peptides, relative to the less active allotypes. This is explained by the fact that MHC ligands with ERAP1-resistant P1 residues are more efficiently generated by a more active variant but are not extensively destroyed even in this context. Increased yields of HLA-B27 ligands by ERAP1 variants with lower activity, relative to more active ones, have been directly demonstrated in vitro (26). 
The molecular weight differences observed among peptides predominant in the context of ERAP1 variants with K528 or R528 were not due to differential peptide length, but to the higher frequency of bulkier P1 and other residues in the more active context. This is consistent with the influence of peptide sequences downstream of the N-terminus on ERAP1 trimming (32) and further indicates an ERAP1 variant-dependent influence of such sequences. Our results suggest that higher occupancy of the substrate-binding site of ERAP1 by bulkier peptides may favor the structural rearrangement required for efficient peptide trimming as a function of residue 528. This is located in an interdomain region that is critical for the conformational transition to the active state $(7,8)$.

Our previous findings in $\mathrm{B}$ 27:04 (13) showed significant differences in the length of HLA-B27 ligands in the distinct ERAP1 contexts examined. There, the most active and the less active ERAP1 allotypes differed by the same mismatches as LG2 and P50, plus Q730E. In contrast, all of the variants in the present study had E730. Thus, the length-dependent effects observed in B*27:04, but not $B * 27: 05$, might be at least partially determined by residue 730 . In vitro experiments using poly-Gly peptides of increasing lengths show that polymorphism at position 528 was not sensitive to substrate length, whereas the relative activity of ERAP1 variants differing at position 730 was substrate length-dependent (Stratikos E: personal communication), which is consistent with our observations.

The substantial effects of the AS-predisposing polymorphism at residue 528 on the HLA-B27 peptidome can influence the biology of HLA-B27 in multiple ways. As previously suggested (31), increased epitope destruction in an active ERAP1 context could render HLA-B27 unable to present certain antigens. This might confer a certain degree of immunodeficiency to HLA-B27-positive individuals that might interfere with the immune control of microorganisms and tumor cells. Increased presentation of a specific tumor antigen upon down-regulation of the ERAP1 ortholog, which leads to improved tumor control, was observed in a murine colorectal carcinoma (33). Yet, the fact that many peptides were expressed in higher amounts in a more active ERAP1 context indicates that increased presentation of other epitopes by HLA-B27 also takes place with AS-predisposing variants.

ERAP1-dependent changes in the HLA-B27 peptidome may also alter $\mathrm{T}$ cell repertoire selection, which is dependent on epitope amounts (34), and modulate the tolerogenic/autoimmune potential of HLA-
B27, affecting the capacity of individuals to respond to, or be tolerized against, particular epitopes even if presented by other MHC molecules. This phenomenon was recently documented in transgenic mice, where coexpression of B7 and B27 resulted in suppression of the HLA-B27-restricted response against a flu-derived epitope through a mechanism involving the negative selection of relevant $\mathrm{T}$ cells (35). Aside from its specifically immunologic effects, ERAP1 polymorphism may affect other peptide-dependent properties of HLA-B27, such as its thermostability (13) or the expression of free heavy chains at the surface of certain cell types (36).

The mechanism of association of ERAP1 with AS is based on altering in a global and significant way the HLA-B27 peptidome through differential effects on the generation/destruction balance of many epitopes, depending on the allelic ERAP1 activity and the $\mathrm{N}$-terminal and other residues of the natural ligands. Due to these alterations, the immunologic and other potentially pathogenic features of HLA-B27 can be substantially affected by ERAP1 polymorphism.

\section{ACKNOWLEDGMENTS}

We thank Sergio Ciordia, Silvia Juarez, and Juan P. Albar (ProteoRed-ISCIII, Centro Nacional de Biotecnología, Madrid, Spain) for help with the mass spectrometry. We also thank Efstratios Stratikos (NCSR Demokritos, Aghia Paraskevi, Greece) for allowing us to cite his unpublished observations.

\section{AUTHOR CONTRIBUTIONS}

All authors were involved in drafting the article or revising it critically for important intellectual content, and all authors approved the final version to be published. Dr. López de Castro had full access to all of the data in the study and takes responsibility for the integrity of the data and the accuracy of the data analysis.

Study conception and design. Sanz-Bravo, López de Castro.

Acquisition of data. Sanz-Bravo, Campos, Mazariegos, López de Castro.

Analysis and interpretation of data. Sanz-Bravo, López de Castro.

\section{REFERENCES}

1. Wellcome Trust Case Control Consortium (WTCC), AustraloAnglo-American Spondylitis Consortium (TASC), Burton PR, Clayton DG, Cardon LR, Craddock N, et al. Association scan of 14,500 nonsynonymous SNPs in four diseases identifies autoimmunity variants. Nat Genet 2007;39:1329-37.

2. Evans DM, Spencer CC, Pointon JJ, Su Z, Harvey D, Kochan G, et al, Australo-Anglo-American Spondyloarthritis Consortium (TASC), Wellcome Trust Case Control Consortium 2 (WTCCC2). Interaction between ERAP1 and HLA-B27 in ankylosing spondylitis implicates peptide handling in the mechanism for HLA-B27 in disease susceptibility. Nat Genet 2011;43:761-7.

3. Brown MA. Breakthroughs in genetic studies of ankylosing spondylitis. Rheumatology (Oxford) 2008;47:132-7.

4. Saric T, Chang SC, Hattori A, York IA, Markant S, Rock KL, et 
al. An IFN- $\gamma$-induced aminopeptidase in the ER, ERAP1, trims precursors to MHC class I-presented peptides. Nat Immunol 2002;3:1169-76.

5. Serwold T, Gonzalez F, Kim J, Jacob R, Shastri N. ERAAP customizes peptides for MHC class I molecules in the endoplasmic reticulum. Nature 2002;419:480-3.

6. Chang SC, Momburg F, Bhutani N, Goldberg AL. The ER aminopeptidase, ERAP1, trims precursors to lengths of MHC class I peptides by a "molecular ruler" mechanism. Proc Natl Acad Sci U S A 2005;102:17107-12.

7. Nguyen TT, Chang SC, Evnouchidou I, York IA, Zikos C, Rock $\mathrm{KL}$, et al. Structural basis for antigenic peptide precursor processing by the endoplasmic reticulum aminopeptidase ERAP1. Nat Struct Mol Biol 2011;18:604-13.

8. Kochan G, Krojer T, Harvey D, Fischer R, Chen L, Vollmar M, et al. Crystal structures of the endoplasmic reticulum aminopeptidase-1 (ERAP1) reveal the molecular basis for $\mathrm{N}$-terminal peptide trimming. Proc Natl Acad Sci U S A 2011;108:7745-50.

9. Strange A, Capon F, Spencer CC, Knight J, Weale ME, Allen MH, et al. A genome-wide association study identifies new psoriasis susceptibility loci and an interaction between HLA-C and ERAP1. Nat Genet 2010;42:985-90.

10. Kirino Y, Bertsias G, Ishigatsubo Y, Mizuki N, Tugal-Tutkun I, Seyahi E, et al. Genome-wide association analysis identifies new susceptibility loci for Behçet's disease and epistasis between HLA-B*51 and ERAP1. Nat Genet 2013;45:202-7.

11. Alvarez-Navarro C, Lopez de Castro JA. ERAP1 structure, function and pathogenetic role in ankylosing spondylitis and other MHC-associated diseases. Mol Immunol 2014;57:12-21.

12. Marcilla M, Lopez de Castro JA. Peptides: the cornerstone of HLA-B27 biology and pathogenetic role in spondyloarthritis. Tissue Antigens 2008;71:495-506.

13. Garcia-Medel N, Sanz-Bravo A, Van Nguyen D, Galocha B, Gomez-Molina P, Martin-Esteban A, et al. Functional interaction of the ankylosing spondylitis-associated endoplasmic reticulum aminopeptidase 1 polymorphism and HLA-B27 in vivo. Mol Cell Proteomics 2012;11:1416-29.

14. Reeves E, Edwards CJ, Elliott T, James E. Naturally occurring ERAP1 haplotypes encode functionally distinct alleles with fine substrate specificity. J Immunol 2013;191:35-43.

15. Zemmour J, Little AM, Schendel DJ, Parham P. The HLA-A,B "negative" mutant cell line C1R expresses a novel HLA-B35 allele, which also has a point mutation in the translation initiation codon. J Immunol 1992;148:1941-8.

16. Calvo V, Rojo S, Lopez D, Galocha B, Lopez de Castro JA Structure and diversity of HLA-B27-specific T cell epitopes: analysis with site-directed mutants mimicking HLA-B27 subtype polymorphism. J Immunol 1990;144:4038-45.

17. Andres AM, Dennis MY, Kretzschmar WW, Cannons JL, Lee-Lin SQ, Hurle B, et al. Balancing selection maintains a form of ERAP2 that undergoes nonsense-mediated decay and affects antigen presentation. PLoS Genet 2010;6:e1001157.

18. Cortes A, Hadler J, Pointon JP, Robinson PC, Karaderi T, Leo P, et al. Identification of multiple risk variants for ankylosing spondylitis through high-density genotyping of immune-related loci. Nat Genet 2013;45:730-8.

19. Paradela A, Garcia-Peydro M, Vazquez J, Rognan D, Lopez de Castro JA. The same natural ligand is involved in allorecognition of multiple HLA-B27 subtypes by a single T cell clone: role of peptide and the MHC molecule in alloreactivity. J Immunol 1998;161:5481-90.

20. Ellis SA, Taylor C, McMichael A. Recognition of HLA-B27 and related antigens by a monoclonal antibody. Hum Immunol 1982;5: 49-59.

21. Paradela A, Alvarez I, Garcia-Peydro M, Sesma L, Ramos M, Vazquez $\mathbf{J}$, et al. Limited diversity of peptides related to an alloreactive T cell epitope in the HLA-B27-bound peptide repertoire results from restrictions at multiple steps along the processing-loading pathway. J Immunol 2000;164:329-37.

22. Garcia-Medel N, Sanz-Bravo A, Barnea E, Admon A, Lopez de Castro JA. The origin of proteasome-inhibitor resistant HLA class I peptidomes: a study with HLA-A*68:01. Mol Cell Proteomics 2012;11:1-15.

23. Hearn A, York IA, Rock KL. The specificity of trimming of MHC class I-presented peptides in the endoplasmic reticulum. J Immunol 2009; 183:5526-36.

24. Harvey D, Pointon JJ, Evans DM, Karaderi T, Farrar C, Appleton $\mathrm{LH}$, et al. Investigating the genetic association between ERAP1 and ankylosing spondylitis. Hum Mol Genet 2009;18:4204-12.

25. Constantino F, Talpin A, Evnouchidou I, Kadi A, Said-Nahal R, Leboime A, et al. Spondyloarthritis-associated polymorphisms of ERAP1 are correlated with gene expression, protein level and enzymatic activity of the amino-peptidase [abstract]. Arthritis Rheum 2013;65 Suppl:S1064.

26. Martin-Esteban A, Gomez-Molina P, Sanz-Bravo A, Lopez de Castro JA. Combined effects of ankylosing spondylitis-associated ERAP1 polymorphisms outside the catalytic and peptide-binding sites on the processing of natural HLA-B27 ligands. J Biol Chem 2014;289:3978-90.

27. Goto Y, Hattori A, Ishii Y, Tsujimoto M. Reduced activity of the hypertension-associated Lys528Arg mutant of human adipocytederived leucine aminopeptidase (A-LAP)/ER-aminopeptidase-1. FEBS Lett 2006;580:1833-8.

28. Garcia-Medel N, Sanz-Bravo A, Alvarez-Navarro C, GomezMolina P, Barnea E, Marcilla M, et al. Peptide handling by HLA-B27 subtypes influences their biological behavior, association with ankylosing spondylitis and susceptibility to endoplasmic reticulum aminopeptidase 1 (ERAP1). Mol Cell Proteomics 2014; 13:3367-80.

29. Evnouchidou I, Kamal RP, Seregin SS, Goto Y, Tsujimoto M, Hattori A, et al. Coding single nucleotide polymorphisms of endoplasmic reticulum aminopeptidase 1 can affect antigenic peptide generation in vitro by influencing basic enzymatic properties of the enzyme. J Immunol 2011;186:1909-13.

30. Chen L, Fischer R, Peng Y, Reeves E, McHugh K, Ternette N, et al. Critical role of endoplasmic reticulum aminopeptidase 1 in determining the length and sequence of peptides bound and presented by HLA-B27. Arthritis Rheumatol 2014;66:284-94.

31. Seregin SS, Rastall DP, Evnouchidou I, Aylsworth CF, Quiroga D, Kamal RP, et al. Endoplasmic reticulum aminopeptidase-1 alleles associated with increased risk of ankylosing spondylitis reduce HLA-B27 mediated presentation of multiple antigens. Autoimmunity 2013;46:497-508.

32. Evnouchidou I, Momburg F, Papakyriakou A, Chroni A, Leondiadis L, Chang SC, et al. The internal sequence of the peptidesubstrate determines its N-terminus trimming by ERAP1. PLoS One 2008;3:e3658.

33. James E, Bailey I, Sugiyarto G, Elliott T. Induction of protective antitumor immunity through attenuation of ERAAP function. J Immunol 2013;190:5839-46.

34. Manz BN, Jackson BL, Petit RS, Dustin ML, Groves J. T-cell triggering thresholds are modulated by the number of antigen within individual T-cell receptor clusters. Proc Natl Acad Sci U S A 2011;108:9089-94.

35. Akram A, Inman RD. Co-expression of HLA-B7 and HLA-B27 alleles is associated with B7-restricted immunodominant responses following influenza infection. Eur J Immunol 2013;43:3254-67.

36. Haroon N, Tsui FW, Uchanska-Ziegler B, Ziegler A, Inman RD. Endoplasmic reticulum aminopeptidase 1 (ERAP1) exhibits functionally significant interaction with HLA-B27 and relates to subtype specificity in ankylosing spondylitis. Ann Rheum Dis 2012; 71:589-95. 